\title{
Hypothyroidism alters mitochondrial morphology and induces release of apoptogenic proteins during rat cerebellar development
}

\author{
R Singh, G Upadhyay and $M$ M Godbole \\ Department of Endocrinology, Sanjay Gandhi Postgraduate Institute of Medical Sciences, Raebareli Road, Lucknow 226014, India \\ (Requests for offprints should be addressed to M M Godbole; Email: godbole@sgpgi.ac.in)
}

\begin{abstract}
Thyroid hormone (TH) deficiency leads to extensive apoptosis during cerebellar development, but the mechanism still remains unclear. Different signals also converge on mitochondria during apoptosis to induce the release of apoptogenic proteins that activate proteolytic cascade through specific enzymes called caspases. Here we studied the effect of hypothyroidism on alterations in mitochondrial structure and translocation of apoptogenic molecules during rat cerebellar development. Structural analysis of mitochondria was studied by electron microscopy. The translocation of apoptogenic molecules was analyzed by Western blotting. TH deficiency led to vacuolization, enlargement and decrease in the number of cristae. The
\end{abstract}

majority of the proapoptotic molecule, Bax, was localized in mitochondria under hypothyroid conditions whereas a limited presence of Bax was detected in the euthyroid state. Translocation of cytochrome $c$, apoptosis-inducing factor (AIF) and second mitochondrial-derived activator of caspases (SMAC) from mitochondria to cytosol was detected primarily in early developmental stages in the hypothyroid condition. These experimental results demonstrate that $\mathrm{TH}$ maintains mitochondrial architecture and inhibits the release of apoptogenic molecules to prevent excess apoptosis during cerebellar development.

Journal of Endocrinology (2003) 176, 321-329

\section{Introduction}

Thyroid hormone (TH) plays an essential role in mammalian brain development. During the critical period of brain development, insufficiency of $\mathrm{TH}$ results in several mental disorders (Schwartz 1983, DeLong et al. 1985, Porterfield \& Hendrich 1993, Oppenheimer \& Schwartz 1997). Hypothyroidism causes severe morphological abnormalities in the entire developing brain, albeit to different extents in different parts of the brain. The size and number of neurons, especially pyramidal neurons in the neocortex, hippocampus and the olfactory bulbs, are reduced. In the cerebellum, TH deficiency has been reported to result in the delayed proliferation and migration of the granule cells from the external to the germinal layer, stunting of the dendritic arborization of the Purkinje cells, diminished axonal myelination and extensive cell loss (Oppenheimer \& Schwartz 1997 and references therein). However the exact mechanism by which hypothyroidism affects brain development remains to be elucidated.

$\mathrm{TH}$ is known to regulate gene expression by binding to its nuclear receptors, expressed in different parts of the brain from early to adult stages (Bernal \& Pekonen 1984, Perez-Castillo et al. 1985, Strait et al. 1991, Bradley et al. 1992, Karmarkar et al. 1993, Chattopadhyay et al.
1995). The influence of $\mathrm{TH}$ on known target genes, namely RC3, MBP, MAP and PCP-2, has been demonstrated but has not increased our understanding of brain development (Silva \& Rudas 1990, Farsetti et al. 1991, Iniguez et al. 1993, Zou et al. 1994). Therefore, several attempts have been made to isolate hitherto unknown tri-iodothyronine $\left(\mathrm{T}_{3}\right)$ regulated genes during brain development by different modern techniques and other model systems (Muñoz et al. 1991, Iglesias et al. 1995, Vega-Nunez et al. 1995, Denver et al. 1997). Using Xenopus as an experimental model, where true $\mathrm{T}_{3}$ negative conditions can be created, 34 cDNAs for TH-regulated genes in the diencephalon were identified. Several of the identified genes were of mitochondrial origin, known to be involved in energy metabolism (Denver et al. 1997). Recently, demonstration of the TH receptor in mitochondria and its involvement in controlling gene expression in mitochondria by different groups further potentiate the role of $\mathrm{TH}$ in energy metabolism (Hashizume \& Ichikawa 1982, Wrutnaik et al. 1995, Enriquez et al. 1999, Rochard et al. 2000). Establishment of the mitochondria's key role in apoptosis, a cardinal feature of neurogenesis, indicates that mitochondria may also play an important role in brain development (Oppenheim 1991, Yuan \& Yanker 2000). However, no correlation has yet been established between mitochondria 
and apoptosis during cerebellar development under hypothyroid conditions.

Divergent cellular stresses including DNA damage, heat shock, oxidative stress and withdrawal of growth factor converge on mitochondria during apoptosis (Adrain \& Martin 2001). Mitochondria contain several apoptosisinducing proteins in the inter-membrane space (Green \& Reed 1998, Hengartner 2001). Bax is a pro-apoptotic gene and is an important regulator of apoptosis. Bax translocation from cytosol to mitochondria is considered to be an important initial event in controlling the release of different apoptogenic molecules (Jurgensmeier et al. 1998). Cytochrome $\mathrm{c}$ is the key executioner of apoptosis and recently other molecules, namely apoptosis-inducing factor (AIF) and second mitochondrial-derived activator of caspases (SMAC), also have been identified (Lorenzo et al. 1999, Daugas et al. 2000, Du et al. 2000, Verhagen et al. 2000). Efflux of cytochrome c in cytosol results in assembly of apoptosome that activates caspase-9. This leads to activation of a proteolytic cascade resulting in cellular disassembly by the effector caspase- 3 . The activated caspase leads to cellular disassembly and DNA fragmentation. AIF, a flavoprotein that resides in the intermembrane space of the mitochondria, translocates to the nucleus during apoptosis. It causes partial chromatin condensation in the periphery of the nucleus by causing degradation of DNA into $>50 \mathrm{~kb}$ fragments through a caspase-independent pathway (Zamzami \& Kroemer 1999). SMAC antagonizes the function of inhibitors of apoptosis (IAPs) present in cytosol that block caspase activation (Daugas et al. 2000, Du et al. 2000, Verhagen et al. 2000).

In the present study, we investigated the effect of hypothyroidism on mitochondrial alterations during rat cerebellar development. Here, we report that TH deficiency leads to structural alterations in mitochondria and enhanced translocation of apoptogenic proteins to cytosol, which results in the enhanced apoptosis during cerebellar neurogenesis observed previously (Xiao \& Nikodem 1998, Singh et al. 2003).

\section{Materials and Methods}

\section{Experimental animals}

Sprague-Dawley rats were housed with a ratio of $12 \mathrm{~h}$ light:12 $\mathrm{h}$ darkness. They had access to chow diet and tap water and were allowed to feed ad libitum. Pregnant rats were divided into two groups $(n=30$ in each group). Hypothyroidism was induced in rats using a previously described protocol (Calvo et al. 1990). Briefly, pregnant dams were given $0.025 \%$ of 2-mercapto-1methylimidazole (MMZ) in drinking water from gestational day 8 and allowed to drink ad libitum. Pups from both hypothyroid and control groups $(n=15$ at each stage) were harvested at postnatal day (P)0, i.e. birth, and thereafter on P4, P8, P12, P16, P20, P24 and P90 (adulthood). At least three pups at each developmental stage from the control and hypothyroid groups were used for all the procedures and each experiment was performed in triplicate. Pups were anesthetized with ether and decapitated. Blood samples were collected when the animals were killed for the determination of TH. All animal procedures performed above were in accordance with the institutional guidelines for animal care and research.

\section{Measurement of serum total $T_{3}\left(T T_{3}\right)$ and total thyroxine $\left(\mathrm{TT}_{4}\right)$}

Serum $\mathrm{TT}_{3}$ and $\mathrm{TT}_{4}$ were measured according to the manufacturer's protocol using standard RIA kits (DPC kit, New York, NY, USA).

\section{Preparation of the cytosolic fraction}

Cerebellum was collected immediately in buffer C (0.32 M sucrose, $1 \mathrm{mM} \mathrm{K-EDTA,} 10 \mathrm{mM}$ Tris-HCl, $\mathrm{pH}$ $7 \cdot 4$ ), homogenized and centrifuged at $1300 \mathrm{~g}$ for $10 \mathrm{~min}$. The supernatant was collected, and the pellet was re-suspended in the same buffer and centrifuged again at $1300 \mathrm{~g}$ for $10 \mathrm{~min}$. The supernatant was pooled and spun at $17000 \mathrm{~g}$ for $15 \mathrm{~min}$ to collect mitochondria. The assay of marker enzymes lactate dehydrogenase (LDH) and cytochrome oxidase (COX) confirmed the supernatant as the cytosolic fraction and the pellet as the mitochondrial fraction. The mitochondrial pellet re-suspended in buffer $\mathrm{C}$ and cytosolic fraction were aliquoted and stored at $-80{ }^{\circ} \mathrm{C}$ for further analysis (Vega-Nunez et al. 1997). The protein concentration was determined by a standard method (Lowry et al. 1951).

\section{Electron microscopy}

Ultrastructural morphology of mitochondria was determined by transmission electron microscopy. Coronal sections of cerebellum of $1 \mathrm{~mm}$ thickness were prepared and fixed rapidly with $4 \%$ paraformaldehyde- $4 \%$ glutaraldehyde in $0.1 \mathrm{M}$ phosphate buffer. After fixation, sections were osmicated in $1 \%$ osmium tetraoxide for $2 \mathrm{~h}$ at $4{ }^{\circ} \mathrm{C}$ and dehydrated in alcohol-acetone series. Tissues were embedded in Araldite resin, and semithin sections $(2.5 \mu \mathrm{m})$ were removed for optical microscopy. Ultrathin sections $(50 \mathrm{~nm})$ were mounted on copper mesh grids and stained with uranyl acetate and lead citrate before examination with a transmission electron microscope (JEOL 1210, Peabody, MA, USA).

\section{Western blotting}

Cytosol and mitochondrial pellet equivalent to $50 \mu \mathrm{g}$ protein having $1 \mathrm{X}$ sample buffer D (10\% glycerol, $2 \%$ SDS, $0.0625 \mathrm{M}$ Tris $-\mathrm{HCl}(\mathrm{pH}$ 6.8)) were heated with 
2-mercaptoethanol at $70{ }^{\circ} \mathrm{C}$ for $7 \mathrm{~min}$. The samples were subjected to $0 \cdot 1 \%$ SDS-15\% PAGE. The gels were run in duplicate; one gel was used for transfer to nitrocellulose membrane while the other was stained with Coomassie blue to confirm equal loading of proteins. The protein was electrotransferred to nitrocellulose membrane and the filter was stained with Ponceau $\mathrm{S}$ to visualize protein bands, to further confirm equal transfer of the proteins.

The nonspecific binding sites were blocked with 5\% BSA in TBST (20 mM Tris- $\mathrm{HCl}(\mathrm{pH} 7 \cdot 4), 137 \mathrm{mM}$ $\mathrm{NaCl}, 0 \cdot 1 \%$ Tween 20 ) for $1 \mathrm{~h}$ at $37^{\circ} \mathrm{C}$. The membranes were washed with $0.1 \%$ BSA in TBST three times at room temperature and were incubated with different primary antibodies (in 1\% BSA in TBST) for $2 \mathrm{~h}$ at room temperature. After washing three times with $0 \cdot 1 \%$ BSA in TBST at room temperature, the membranes were incubated with secondary antibody labeled with horseradish peroxidase for $2 \mathrm{~h}$ at room temperature. The filter was washed three times with $0 \cdot 1 \%$ BSA in TBST (Sambrook et al. 1989). The color was developed by immuno-Blot Assay kits (Bio Rad, Hercules, CA, USA). Each experiment was repeated three times independently.

\section{Statistical analysis}

The relative amount of each protein was determined quantitatively. Blots from all the three replicates of control and hypothyroid groups were analyzed microdensitometrically using an Alpha Imager (Alpha Imager Corp., San Leandro, CA, USA). The standard error of the mean between the groups was analyzed by SPSS software (version 9.0) using Levene's independent sample $t$ test. Ontogenic expression of each protein was assessed by one-way ANOVA and data were further analyzed for specific changes over time using Tukey's $B$ test.

\section{Results}

\section{Severe hypothyroidism is induced by $M M Z$}

Hypothyroid pups showed stunted growth and sluggish behavior as compared with controls. The creeping and opening of eyes was delayed, especially from stages $\mathrm{P} 4$ to P16. Serum $\mathrm{TT}_{4}$ and $\mathrm{TT}_{3}$ levels of growing pups increased with age whereas they decreased significantly $(P<0 \cdot 005, P<0 \cdot 05)$ in MMZ-treated groups and were hardly detectable in hypothyroid rats (Fig. 1).

\section{TH deficiency alters mitochondrial structure in developing cerebellum: electron microscopic analysis}

To assess whether TH deficiency during cerebellar development results in structural alteration, electron microscopy was performed at stage P12, where maximum apoptosis and caspase activity was seen previously (Singh et al. 2003).
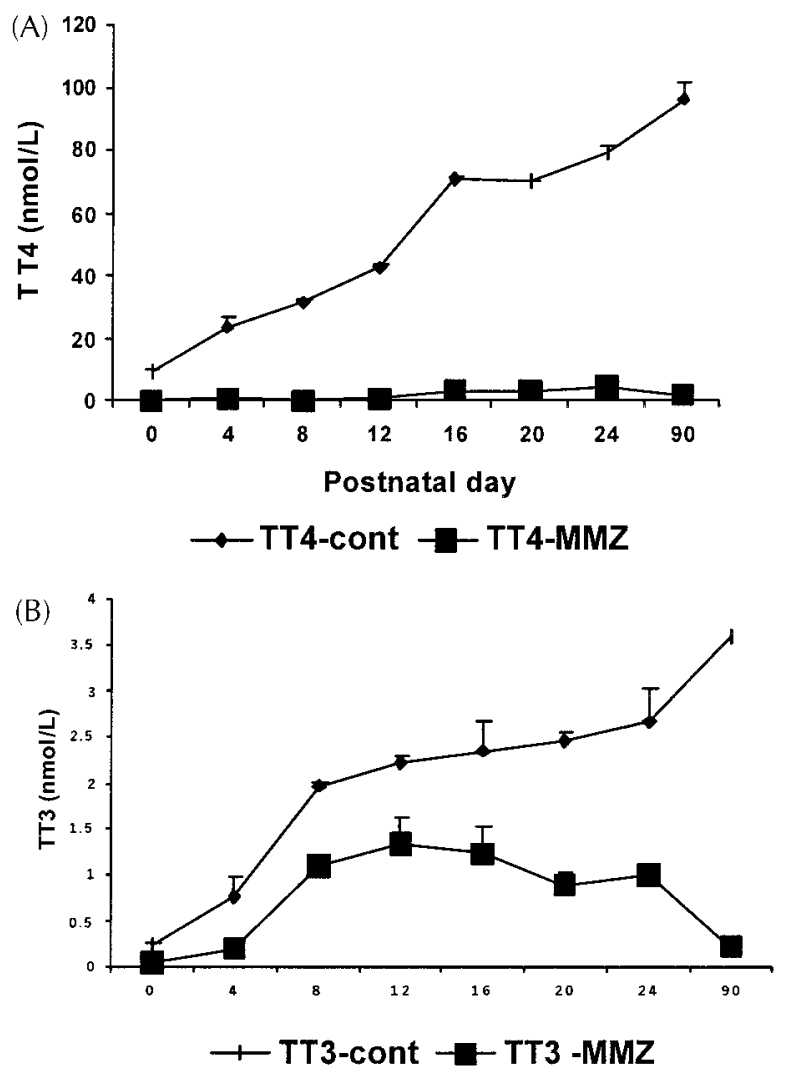

Figure 1 Serum $\pi_{3}$ and $\pi_{4}$ in developing pups. Serum from pups of control and hypothyroid rats were analyzed for $\Pi_{3}$ and $\Pi_{4}$ from postnatal day 0 to 90 days. The blood was collected from five of each stage and from three different groups of control and MMZ-treated pups. Serum $\mathrm{TT}_{4}(\mathrm{~A})$ and $\mathrm{TT}_{3}(\mathrm{~B})$ were determined by RIA. The results are expressed as means \pm S.E.M.

It has been observed previously that the programmed cell death after birth is limited to the internal germinal layer (IGL) of cerebellum although some apoptotic cells are also observed in the external granular layer (EGL) (Xiao \& Nikodem 1998). Therefore, sections from the IGL were processed for electron microscopy to see the alteration in mitochondrial morphology. The mitochondria from euthyroid cerebellum presented with highly packed inner membranes and many visible cristae characteristic of normal morphology (Fig. 2A). Altered mitochondrial morphology with enlarged size, increased vacuolization and a decrease in the number of cristae was seen under hypothyroid conditions. The number of mitochondria having altered morphology also increased under hypothyroid conditions (Fig. 2B).

Bax is translocated to mitochondria during hypothyroid conditions

Bax translocation from cytosol to mitochondria is an important event that may induce structural change in 
A

Control

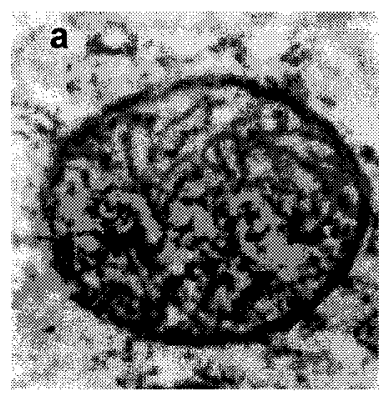

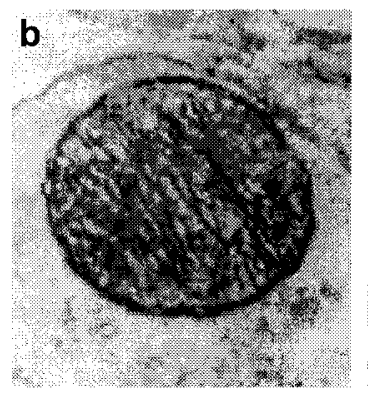

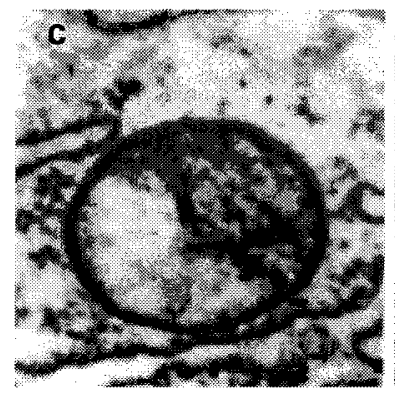

Hypothyroid

B

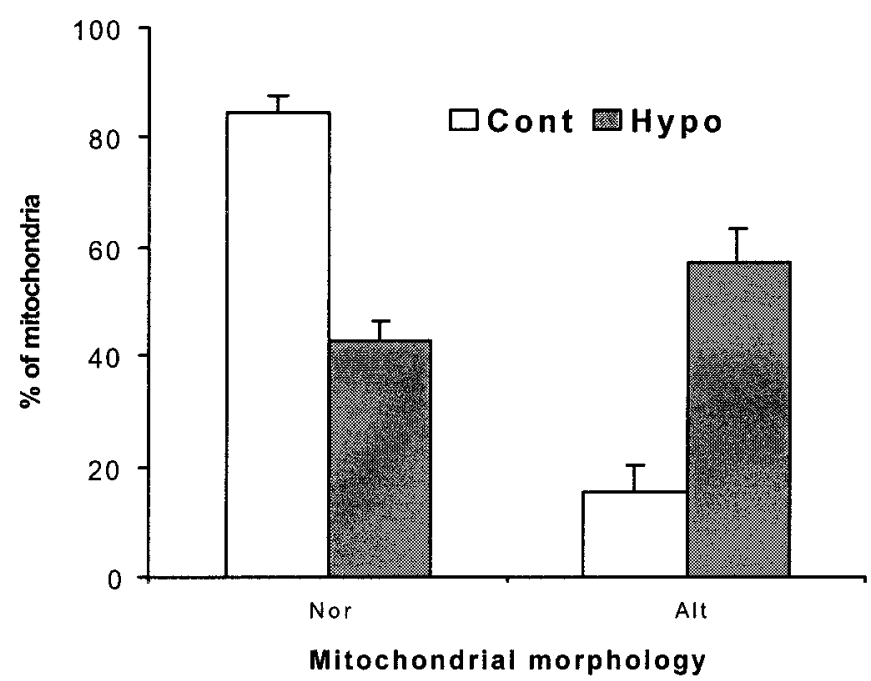

Figure 2 Structural analysis of mitochondria in developing cerebellum. (A) The coronal sections of cerebellum from the control $(a, b)$ and hypothyroid pups (c, d) at the P12 stage were analyzed using electron microscopy for the mitochondrial morphology $(20000 \times)$. (B) Quantitative analysis of altered mitochondrial structure. The number of mitochondria having normal and altered structure was counted in five different views. The results were expressed from five different views from each slide of three different groups each of control and MMZ-treated pups. The results are expressed as means \pm S.E.M.

mitochondria (Jurgensmeir et al. 1998). To see whether ultrastructural changes are due to translocation of $B a x$ under hypothyroid conditions, Western blot analysis of both mitochondrial pellet and cytosol was performed. In euthyroid conditions, a $21 \mathrm{kDa}$ protein was detected in mitochondria as well as in the cytosol (Fig. 3A). Bax was expressed constitutively from $\mathrm{P} 0$ to $\mathrm{P} 16$. The expression of $B a x$ varied with age $\left(F_{7,24}=170\right)$ and increased significantly at P20 to adult stage $(P<0 \cdot 001)$. The localization of Bax in the mitochondrial pellet did not show any significant variation (Fig. 3A,B). However, under hypothyroid conditions, expression of Bax was higher in cytosol at the P0 stage then decreased significantly $(P<0 \cdot 001)$ from
P4-P24 and adult, with a concomitant increase in the mitochondrial pellet. The presence of $B a x$ in the mitochondrial fraction was significantly higher $(P<0 \cdot 001)$ as compared with the euthyroid state at all stages of development (Fig. 3C,D).

\section{Hypothyroidism induces cytochrome c release from mitochondria} to cytosol

Cytochrome $\mathrm{c}$ is the principal initiator molecule released from mitochondria to cytosol to initiate the apoptotic program in the cell (Green \& Reed 1998). Therefore, we studied cytochrome c translocation. In the euthyroid state, 
Control
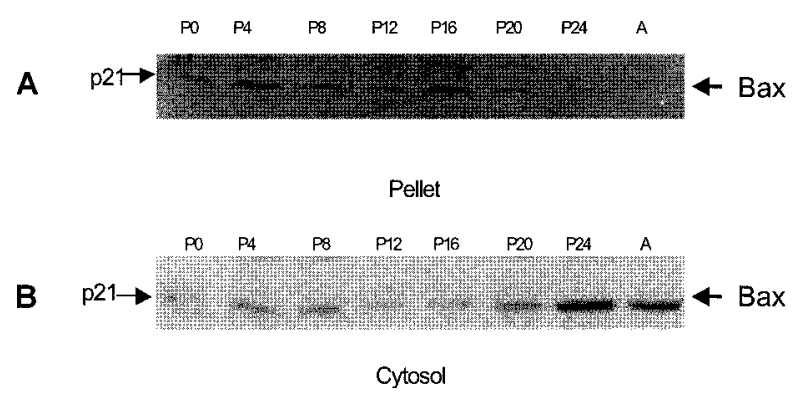

Hypothyroid

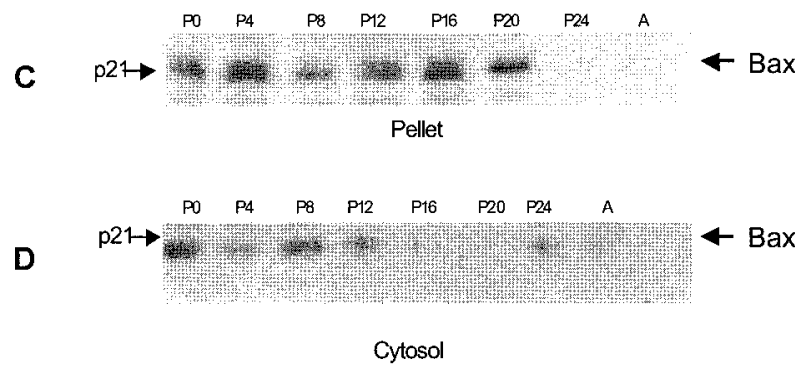

Figure 3 Analysis of translocation of Bax from cytosol to mitochondria. The mitochondrial pellet and cytosol were isolated from developing cerebellum (P0, P4, P8, P12, P16, P20, P24 and adult (A)) of control (A and B) and hypothyroid (C and D) pups. Cytosolic and mitochondrial proteins $(50 \mu \mathrm{g})$ were subjected to $15 \%$ SDS-PAGE and analyzed by Western blot using rabbit polyclonal antibody. Both the experiments were performed at the same time to compare the intensity of Bax present in the pellet and in the cytosol. The experiments were repeated three times.

the $20 \mathrm{kDa}$ protein corresponding to cytochrome $\mathrm{c}$ was detected in the mitochondrial pellet (Fig. 4A) but not in the cytosolic fraction (Fig. 4B). However, the decreased intensity of the band for cytochrome $c$ was observed in the mitochondrial pellet at P0 and P8 (Fig. 4A) but its corresponding presence was not detected in the cytosol. Conversely, in the hypothyroid condition, a significantly higher level $(P<0 \cdot 001)$ of cytochrome $\mathrm{c}$ was detected in the cytosol at the early developmental stage from P0 to P8 and the decreased intensity was observed in the mitochondrial pellet $(P<0 \cdot 001)$. However, at late stages of development, enhanced intensity of the cytochrome $\mathrm{c}$ band was observed in the mitochondrial pellet, with a concomitant decrease in the cytosol (Fig. 4C,D).

\section{Hypothyroidism induces release of AIF from mitochondria}

AIF is released from mitochondria to initiate caspaseindependent apoptosis (Lorenzo et al. 1999). We also studied hypothyroidism-induced release of AIF from mitochondria during cerebellar development. In the
A

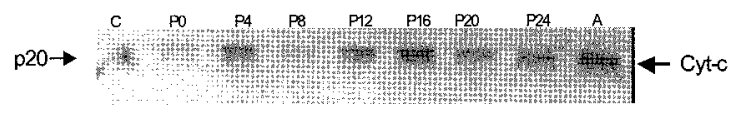

Pellet

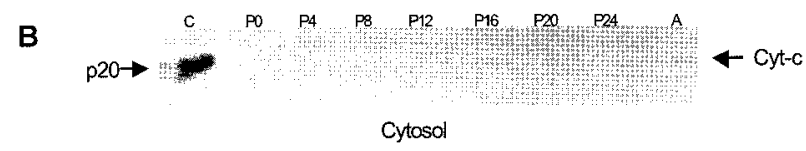

Cytosol

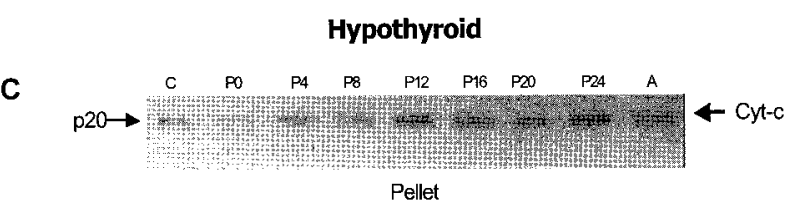

D

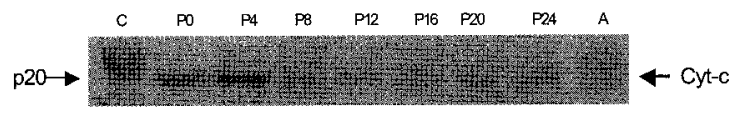

Cytosol

Figure 4 Analysis of cytochrome c translocation. The mitochondrial pellet and cytosol were isolated from developing cerebellum (P0, P4, P8, P12, P16, P20, P24 and adult (A)) of control ( $\mathrm{A}$ and $\mathrm{B}$ ) and hypothyroid $(\mathrm{C}$ and $\mathrm{D})$ pups. Cytosolic and mitochondrial proteins $(50 \mu \mathrm{g})$ were subjected to $15 \%$ SDS-PAGE and analyzed by Western blot. Both the experiments were performed at the same time and repeated three times.

euthyroid state, Western blot analysis showed a $58 \mathrm{kDa}$ protein corresponding to AIF in the mitochondrial pellet but not in cytosol (Fig. 5A,B). However, under hypothyroid conditions, low expression of AIF was detected in the mitochondrial pellet, and most of the AIF was detected in the cytosolic fraction. It was significantly $(P<0 \cdot 001)$ high as compared with the euthyroid condition at all the developmental stages except P20 and the adult stage (Fig. 5C,D).

\section{SMAC translocates from mitochondria to cytosol during development}

The release of SMAC from mitochondria inhibits IAPs present in cytosol, making the cell competent to undergo apoptosis (Daugas et al. 2000). Western blot analysis of cerebellar cellular components under euthyroid conditions revealed the presence of a $\sim 32 \mathrm{kDa}$ protein corresponding to SMAC in the cytosol from P0 to P16 that decreased $\left(F_{7,24}=199 \cdot 0\right)$ at later stages in euthyroid conditions (Fig. $6 \mathrm{~B})$. A significantly lower level of SMAC was detected in the mitochondrial pellet except at P16 and P20 where significantly higher $(P<0 \cdot 001)$ expression was observed (Fig. 6A). In hypothyroid conditions, the expression of SMAC was significantly higher $(P<0 \cdot 001)$ as compared 


\section{Control}

A

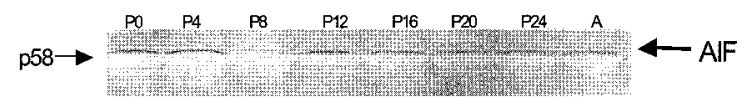

Pellet

B

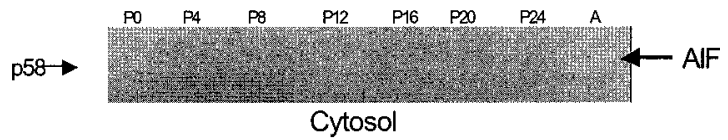

Cytosol

Hypothyroid

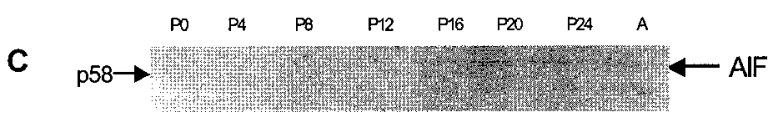

Pellet

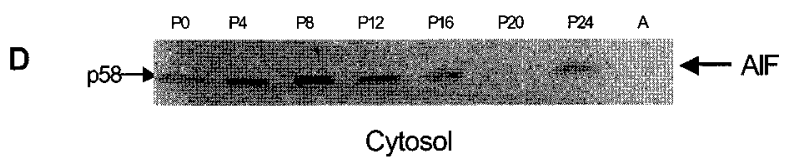

Figure 5 Analysis of AIF translocation to cytosol. The mitochondrial pellet and cytosol were isolated from developing cerebellum (P0, P4, P8, P12, P16, P20, P24 and adult (A)) of control $(\mathrm{A}$ and $\mathrm{B})$ and hypothyroid $(\mathrm{C}$ and $\mathrm{D})$ pups. Cytosolic and mitochondrial proteins $(50 \mu \mathrm{g})$ were subjected to $15 \%$ SDS-PAGE and were analyzed by Western blot using goat polyclonal antibody. Both the experiments were performed at the same time and repeated three times.

with control pups and it varied with age (Fig. 6C). Significantly $(P<0 \cdot 001)$ high levels of SMAC were observed in cytosol from P0 to P16 and was undetectable thereafter, with a concomitant increase in mitochondria (P20-P24 and adult) (Fig. 6D).

\section{Discussion}

Here we report that TH deficiency causes alteration in mitochondrial morphology, leading to translocation of apoptogenic molecules located in inter-membrane space to cytosol during cerebellar development. This may lead to extensive apoptosis during cerebellar neurogenesis, leading to a decrease in cell number during hypothyroid conditions, as observed previously.

We used the rat model to understand the effect of $\mathrm{TH}$ on mitochondrial-mediated apoptosis during cerebellar development. The cerebellar development is largely postnatal and various anatomical alterations induced by $\mathrm{TH}$ deficiency have been well documented and mimic the condition in humans (Koibuchi \& Chin 2000). The pups of dams on MMZ were severely hypothyroid, as indicated by significantly low serum TH levels (Fig. 1).
Control

A

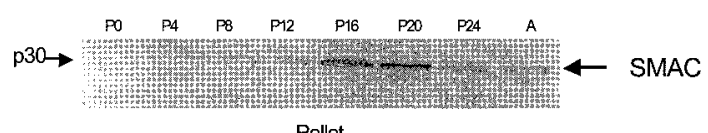

Pellet

B

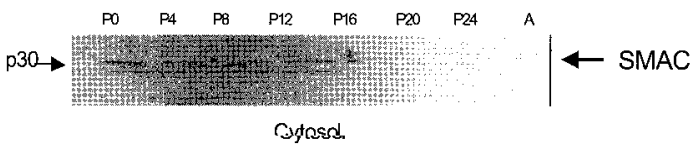

Hypothyroid

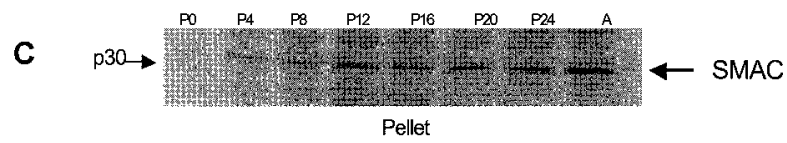

D

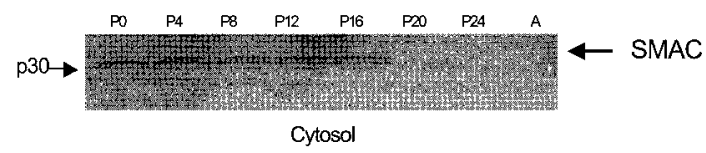

Figure 6 Analysis of SMAC release from mitochondria to cytosol. The mitochondrial pellet and cytosol were isolated from developing cerebellum (P0, P4, P8, P12, P16, P20, P24 and adult (A)) of control (A, B) and hypothyroid (C, D) pups. Cytosolic and mitochondrial proteins $(50 \mu \mathrm{g})$ were subjected to $15 \%$ SDS-PAGE and analyzed by Western blot using rabbit polyclonal antibody. Both the experiments were performed at the same time and repeated three times.

Ultrastructural analysis of mitochondria from the IGL from cerebellum revealed that under euthyroid conditions, morphological and structural integrity was maintained whereas in hypothyroid conditions, volume dysregulation, decrease in number of cristae and vacuolization was observed (Fig. 2). However, the mitochondrial membrane remained intact under both the conditions, suggesting that the alteration may be due to the loss of transmembrane potential (Green \& Reed 1998). Similar findings were also reported in developing cerebral cortex under hypothyroid conditions (Vega-Nunez et al. 1997).

The alteration in mitochondrial morphology in hypothyroid conditions may be due to the translocation of apoptogenic molecules. Translocation of Bax to mitochondrial and other membrane sites and triggering of catastrophic transformation of mitochondrial function in response to death signals has been shown in vitro (Goping et al. 1998, Krajewski et al. 1999, Cao et al. 2001, Ghribi et al. 2001, Yamaguchi et al. 2001). In euthyroid conditions, we found that Bax was predominantly localized in mitochondria during early developmental stages and decreased with age. Previously, we observed the high expression of $B c l-2$ and $B c l-x_{L}$ under euthyroid conditions (Singh et al. 2003). It is reasonable to assume that 
those constitutively expressed anti-apoptotic molecules, namely Bcl-2 and Bcl- $\mathrm{x}_{\mathrm{L}}$, probably heterodimerize with Bax to regulate the mitochondrial volume as proposed previously (Green \& Reed 1998, Adrain \& Martin 2001). Hypothyroidism leads to increased translocation of Bax from cytosol to mitochondria. The reduced expression of Bcl-2 and Bcl- $\mathrm{x}_{\mathrm{L}}$ reported previously (Singh et al. 2003) probably prevents the required heterodimerization necessary for maintaining the membrane potential and mitochondrial volume. To our knowledge, this is the first demonstration of translocation of Bax during cerebellar neurogenesis under hypothyroid conditions. Recently, immortalized neuronal precursor cells have been shown to undergo apoptosis upon serum withdrawal, where translocation of Bax to mitochondria plays an important role in the initiation of apoptosis (Colombaioni et al. 2002). Hypothyroidism is also known to reduce nerve growth factor (NGF) in all parts of the brain, including the cerebellum, specifically during the early postnatal period (P4-P8) (Figueiredo et al. 1993, Alvarez-Dolado et al. 1994). Thus, withdrawal of NGF and other neurotrophic factors during hypothyroid conditions may activate mitochondrialmediated apoptosis through translocation of Bax.

Hypothyroidism-induced mitochondrial structural changes and translocation of Bax observed here suggest a strong possibility of the release of apoptogenic proteins from mitochondria to initiate the downstream apoptotic pathways. Mitochondria are known to harbor several molecules, namely cytochrome c, AIF and SMAC, that once released to cytosol activate the caspase-dependent and -independent pathways. Therefore we studied the translocation of apoptogenic molecules from mitochondria to cytosol. In euthyroid conditions, cytochrome $\mathrm{c}$ was detected in mitochondria at all the developmental stages, but was undetectable in cytosol (Fig. 4). However, hypothyroidism-induced cytochrome $\mathrm{c}$ release to cytosol during early development may contribute to initiation of apoptosis through formation of apoptosomes and activating a caspase cascade that may result in enhanced apoptosis (Green \& Reed 1998).

Limited release of cytochrome c only partially explains the extensive apoptosis seen under hypothyroid conditions. Hence, we also investigated the translocation of other known apoptogenic molecules. AIF translocates from mitochondria to the nucleus, where it causes peripheral chromatin disintegration by a caspase-independent pathway (Zamzami \& Kroemer 1999). The presence of adequate levels of TH prevent the release of AIF from mitochondria to cytosol, as AIF was not detected in the cytosol in euthyroid conditions (Fig. 5). We observed that hypothyroidism induces the translocation of AIF from mitochondria to cytosol during the early developmental period ( $\mathrm{P} 0-\mathrm{P} 12)$ to activate the caspase-independent pathway of apoptosis during cerebellar development.

In response to apoptotic stimuli, SMAC is released in cytosol and binds to the IAPs, thereby relieving the IAP inhibition of caspases (Deveraux \& Reed 1999). It is known that an isoform of IAP known as NIAP (neural IAP) is confined to the neurons at high levels, preventing the apoptosis from the different physiological and pathological insults (Kuida et al. 1998). However during neurogenesis, due to positive selection of neurons making appropriate connections, extra-synaptic cells die by programmed cell death. The competency to undergo programmed cell death may be achieved by the release of mitochondrial SMAC during early development (P0-P16) observed here (Fig. 6). The higher levels of SMAC in cytosol under hypothyroid conditions reflect the fact that large numbers of neuronal cells achieve competency to undergo apoptosis. The absence of SMAC in adult cerebellum reported here is in agreement with earlier studies and also in consonance with steady state neurogenesis observed in adults (Du et al. 2000, Verhagen et al. 2000). The mitochondria from adult cerebellum seem to be refractory to apoptotic stimuli as limited translocation of apoptogenic molecules to cytosol is observed both in hypo- and euthyroid conditions. The recalcitrant nature of adult cerebellum has also been reported previously (Deshmukh \& Jhonson 1998).

In conclusion, this study shows for the first time that the presence of adequate levels of TH maintains mitochondrial integrity and physiology to inhibit excess apoptosis by preventing the release of apoptogenic proteins during cerebellar neurogenesis. However, its deficiency leads to morphological alteration resulting in translocation of apoptogenic molecules from mitochondria. The mechanism of release of apoptogenic molecules from mitochondria, extensive apoptosis and its effect on the manifestation of neuronal disorders need further study. The present results do not undermine the nuclear action of $\mathrm{TH}$ but suggest that $\mathrm{TH}$ is an important regulator in maintaining the coordination between mitochondria and nuclei, at least in controlling the process of apoptosis, however further experimentation is needed.

\section{Acknowledgements}

This work constitutes the $\mathrm{PhD}$ thesis of Rajesh Singh. The CSIR Fellowship to Rajesh Singh is deeply acknowledged. We are highly grateful to Dr X Wang (University of Southwestern Texas Medical Center, Dallas, TX, USA) for the kind gift of antibodies for SMAC and caspase-9, and to Dr R Jemmerson (University of Minnesota, USA) for cytochrome $\mathrm{c}$ antibodies. The authors wish to acknowledge Mr Manoj Shukla and Mr Vivek Saigal for their technical assistance, and $\mathrm{Mr}$ Ashwani Mishra and Mr Pankaj Mishra for statistical analysis. The help of Ashok Kumar in carrying out a major revision and critical reading of the manuscript is gratefully acknowledged. 


\section{References}

Adrain C \& Martin SJ 2001 The mitochondrial apoptosome: a killer unleashed by the cytochrome c. Trends in Biochemical Sciences $\mathbf{2 6}$ 390-397.

Alvarez-Dolado M, Iglesais T, Rodriguez-Pena A, Bernal J \& Munoz A 1994 Expression of neurotrophins and the trk family receptors in normal and hypothyroid rat brain. Molecular Brain Research 27 249-257.

Bernal J \& Pekonen F 1984 Ontogenesis of the nuclear $3,5,3^{\prime}$-triiodothyronine receptor in the human fetal brain. Endocrinology 114 677-679.

Bradley DJ, Towle HC, Young HC \& Young WS 1992 Spatial and temporal expression of $\alpha$ and $\beta$ thyroid hormone receptor mRNAs, including the $\beta 2$ subtype, in developing mammalian nervous system. Journal of Neuroscience 12 2288-2302.

Calvo R, Obregon MJ, Ruiz de Ona C, Escobar del Rey F \& Morreale de Escobar G 1990 Congenital hypothyroidism, as studied in rats. Critical role of maternal thyroxine but not of 3,5,3' triiodothyronine in the protection of the fetal brain. Journal of Clinical Investigation 86 889-899.

Cao G, Minamai M, Pai W, Yan C, Chan D, O’Horn C, Graham SH \& Chen J 2001 Intracellular Bax translocation after transient cerebral ischemia: implications for a role of the mitochondrial apoptotic signaling pathway in ischemic neuronal death. Journal of Cerebral Blood Flow and Metabolism 21 321-333.

Chattopadhyay N, Kher R, Virmani J \& Godbole MM 1995 Differential expression of alpha and beta thyroid hormone receptor genes in the developing rat brain under hypothyroidism. Biology of the Neonate 67 64-71.

Colombaioni L, Colombini L \& Garcia-Gill M 2002 Role of mitochondria in serum withdrawal induced apoptosis of immortalized neuronal precursors. Developmental Brain Research 134 93-102.

Daugas E, Nochy D, Ravagnan L, Loeffler M, Susin SA, Zamzami N \& Kroemer G 2000 Apoptosis-inducing factor (AIF): a ubiquitous mitochondrial oxidoreductase involved in apoptosis. FEBS Letters 476 118-123.

DeLong GR, Stanburry JB \& Fierro-Benitez R 1985 Neurological signs in congenital iodine deficiency disorders (endemic cretinism). Developmental Medicine and Child Neurology 27 317-324.

Denver RJ, Pavgi S \& Shi YB 1997 Thyroid hormone-dependent gene expression program for Xenopus neural development. Journal of Biological Chemistry 272 8179-8188.

Deshmukh M \& Jhonson EM 1998 Evidence of novel event during neuronal death: development of competence-to-die in response to cytoplasmic cytochrome c. Neuron 21 695-705.

Deveraux QL \& Reed JC 1999 IAP family of proteins - suppressors of apoptosis. Genes and Development 13 239-252.

Du C, Fang M, Li Y, Li L \& Wang X 2000 Smac, a mitochondrial protein that promotes cytochrome c-dependent caspase activation by eliminating IAP inhibition. Cell 102 33-42.

Enriquez JA, Fernandez-Silva P, Garrido-Perez N, Lopez-Perez MJ, Perez-Martos A \& Montoya J 1999 Direct regulation of mitochondrial RNA synthesis by thyroid hormone. Molecular and Cellular Biology 19 657-670.

Farsetti A, Robbins J \& Nikodem V 1991 Molecular basis of thyroid hormone regulation of mylein basic protein gene expression in rodent brain. Journal of Biological Chemistry 266 23226-23232.

Figueiredo BC, Otten U, Strauss S, Volk B \& Maysingrr D 1993 Effect of hypo and hyperthyroidism on the levels of nerve growth factor and its low affinity receptor in cerebellum. Developmental Brain Research 72 237-244.

Ghribi O, Hermann MM, Forbes MS, Dewitt DA \& Savory J 2001 GDNF protects against aluminum induced apoptosis in upregulating $\mathrm{Bcl}-2$ and $\mathrm{Bcl}-\mathrm{x}_{\mathrm{L}}$ and inhibiting mitochondrial translocation. Neurobiology Disorders 8 764-773.
Goping IS, Gross A, Lavoie JN, Nguyen M, Jemmerson R, Roth K, Korsmeyer SJ \& Shore GC 1998 Regulated targeting of Bax to mitochondria. Journal of Cell Biology 143 207-215.

Green DR \& Reed JC 1998 Mitochondria and apoptosis. Science 281 1309-1311.

Hashizume K \& Ichikawa K 1982 Localization of 3,5,3 tri-iodothyronine receptor in rat mitochondrial membrane. Biochemical and Biophysical Research Communications 106 920-926.

Hengartner MO 2000 The biochemistry of apoptosis. Nature $\mathbf{4 0 7}$ 770-776.

Iglesias T, Caubin J, Zaballos A, Bernal J \& Munoz A 1995 Identification of the mitochondrial NADH dehydrogenase subunit 3 (ND3) as a thyroid hormone regulated gene by whole genome PCR analysis. Biochemical and Biophysical Research Communications 210 995-1000.

Iniguez MA, Rodriguez-Pena A, Ibarolla N, Aguilera M, Munoz A \& Bernal J 1993 Thyroid hormone regulation of RC3, a brain-specific gene encoding a protein kinase-C substrate. Endocrinology 133 467-473.

Jurgensmeier J, Xie Z, Deveraux Q, Ellerby L, Bredesen D \& Reed JC 1998 Bax directly induces release of cytochrome $\mathrm{c}$ from isolated mitochondria. PNAS 95 4997-5002.

Karmarkar MG, Prabhakaran D \& Godbole MM 1993 5'-Monodeiodinase activity in developing human cerebral cortex. American Journal of Clinical Nutrition Supplement 57 291S-294S.

Koibuchi N \& Chin WW 2000 Thyroid hormone action and brain development. Trends in Endocrinology and Metabolism 11 123-128.

Krajewski S, Krajewska M, Ellerby LM, Welsh K, Xie Z, Deveraux QL, Selvesen GS, Bredsen DE, Rosenthal RE, Fiskum G \& Reed JC 1999 Release of caspase-9 from mitochondria during neuronal apoptosis and cerebral ischemia. PNAS 96 5752-5757.

Kuida K, Haydar TF, Kaunc Y, Gu Y, Taya C, Karasuyama H, Su MS, Rakic P \& Flavell RA 1998 Reduced apoptosis and cytochrome c-mediated caspase activation in mice lacking caspase 9. Cell 94 325-337.

Lorenzo H, Susin SA, Peninger L \& Kroemer G 1999 Apoptosis Inducing Factor: a phylogenetically old caspase independent effector of cell death. Cell Death and Differentiation 6 516-524.

Lowry OH, Rosebrough NH, Farr AL \& Randall RJ 1951 Protein measurements with the Folin phenol reagent. Journal of Biological Chemistry 193 265-275.

Muñoz A, Rodriguez-Péna A, Perez-Castillo A, Ferreiro B, Sutcliffe JG \& Bernal J 1991 Effects of neonatal hypothyroidism on rat brain gene expression. Molecular Endocrinology 5 273-280.

Oppenheim RW 1991 Cell death during development of the nervous system. Annual Review of Neuroscience 14 453-501.

Oppenheimer JH \& Schwartz HL 1997 Molecular basis of thyroid hormone-dependent brain development. Endocrine Reviews 18 $462-475$.

Perez-Castillo A, Bernal J, Ferreiro B \& Pans T 1985 The early ontogenesis of thyroid hormone receptor in rat fetus. Endocrinology 117 2457-2461.

Porterfield SP \& Hendrich CE 1993 The role of thyroid hormones in prenatal and neonatal neurological developmental - current perspectives. Endocrine Reviews 14 94-106.

Rochard P, Rodier A, Casa F, Cassar-Malek I, Marcha-Victorian S, Daury L, Wrutnaik C \& Cabello G 2000 Mitochondrial activity is involved in the regulation of myoblast differentiation through myogenin expression and activity of myogenic factors. Journal of Biological Chemistry 275 2733-2744.

Sambrook J, Fritsch EF \& Maniatis J 1989 Molecular Cloning: $A$ Laboratory Manual, edn 2. Cold Spring Harbor, NY: Cold Spring Harbor Laboratory.

Schwartz HL 1983 Effect of thyroid hormone in growth and development. In Molecular Basis of Thyroid Hormone Action, pp 413-444. Eds JH Oppenheimer \& HH Samuels. New York: Academic Press. 
Silva JE \& Rudas P 1x990 Effects of congenital hypothyroidism on microtubule-associated protein expression in the cerebellum of the rat. Endocrinology 126 1276-1282.

Singh R, Upadhyay G, Kumar S, Kapoor A, Kumar A, Tiwari M \& Godbole MM 2003 Hypothyroidism alters the expression of Bcl-2 family genes to induce enhanced apoptosis in developing cerebellum. Journal of Endocrinology 146 39-46.

Strait KA, Schwartz HL, Seybold VS, Ling NC \& Oppenheimer JH 1991 Immunofluorescent localization of thyroid hormone receptor proteins $\beta 1$ and variant $\alpha 2$ in selected tissues: cerebellar Purkinje cells as a model for $\beta 1$ receptor mediated developmental effects of thyroid hormone in brain. PNAS 88 3887-3891.

Vega-Nunez E, Menendez-Hurtado A, Garesse R, Santos A \& Perez-Castillo A 1995 Thyroid hormone regulated brain mitochondrial genes revealed by differential cDNA cloning. Journal of Clinical Investigation 96 893-899.

Vega-Nunez E, Alaverz AM, Menendez-Hurtado A, Santos A \& Perez-Castillo A 1997 Neuronal mitochondrial morphology and transmembrane potential are severely altered by hypothyroidism during rat brain development. Endocrinology 138 3771-3778.

Verhagen AM, Ekert PG, Pakusch M, Silke J, Connolly LM, Reid GE, Moritz RL, Simpson RJ \& Vaux DL 2000 Identification of DIABLO: a mammalian protein that promotes apoptosis by binding and antagonizing IAP proteins. Cell 102 43-53.
Wrutnaik CC, Cassar-Malek I, Marchal S, Rascle A, Heusser S, Keller JM, Felchon J, Dauca M, Samarut J, Ghysdael J \& Cabello G 1995 A $43 \mathrm{kDa}$ protein related to c-Erb A- $\alpha 1$ is located in the mitochondrial matrix of rat liver. Journal of Biological Chemistry 270 16347-16354.

Xiao Q \& Nikodem V 1998 Apoptosis in the developing cerebellum of the thyroid hormone deficient rat. Frontiers in Bioscience 3 52-57.

Yamaguchi A, Tamami M, Matsuzaki H, Namikawa K, Kiyama H, Mitsuda N \& Tohyama M 2001 Akt activation protects hippocampal neurons from apoptosis inhibiting transcriptional activity of p53. Journal of Biological Chemistry 276 5256-5264.

Yuan J \& Yanker BA 2000 Apoptosis in the nervous system Nature 407 802-808.

Zamzami N \& Kroemer G 1999 Condensed matter in cell death. Nature 410 127-128.

Zou L, Hagen SG, Strait KA \& Oppenheimer JH 1994 Identification of thyroid hormone response elements in rodent Pcp-2, a developmentally regulated gene of cerebellar Purkinje cells. Journal of Biological Chemistry 269 13346-13352.

Received in final form 20 November 2002 Accepted 9 December 2002 